\title{
Evaluation of the Friedmann Visual Field Analyser Mark II. Part 2. Results from a population with induced visual field defects
}

\author{
DAVID B. HENSON AND SANDRA M. DIX \\ From the Department of Optometry at the University of Wales Institute of Science and Technology
}

SUmmary The Friedmann Visual Field Analyser Mark II is evaluated with a group of 301 normal eyes, 155 of which had an induced visual field defect. Measures of sensitivity and specificity are calculated from these data by criteria similar to those used by other researchers. The inadequacies of using pass/fail criteria in the clinical situation are discussed, and a solution is proposed in which each field response is scored and the score compared with those from normal and defective populations. An example is given of how this form of analysis could be incorporated into the clinical situation.

One of the most useful ways of evaluating a piece of visual field equipment is to calculate its sensitivity (what percentage of defects it detects) and its specificity (what percentage of normal subjects it fails). These two measures are normally obtained from a clinical trial in which two populations are examined, one with visual field defects and one without. Finding a population of patients with precisely defined visual field defects is a major problem in these types of trials. The recognition of a defect can be made only through visual field examination, and thus an evaluation of a piece of visual field equipment, in essence, becomes a comparison between one piece of equipment/technique and another. If the evaluated piece of equipment is compared with one which has a low sensitivity, it will appear to be more sensitive than if it were compared to one which has a high sensitivity. Many researchers have attempted to overcome this problem by performing on every patient a very extensive visual field examination with the hope that all defects will be detected. ${ }^{1}{ }^{3}$ While this is to be commended as an attempt to solve one problem, it does mean that the patient sample is invariably biased. Not all patients can be relied upon to give accurate results throughout an examination that may extend to over 1 hour/eye. Any evaluation that is confined to these 'super' patients is likely to lead to better results than can be expected from a normal population.

Correspondence to D. B. Henson PhD. Department of Optometry. UWIST, Colum Drive, Cardiff CF1 3EU.
In this paper we report on an evaluation of the Friedmann Visual Field Analyser Mark II (VFA), ${ }^{4}$ in which the problems involved in finding a population of patients with precisely defined defects have been overcome. In this evaluation visual field defects have been induced, via a modification of the equipment, in normal patients. Because these defects are induced their location and depth are precisely known without each patient having to be submitted to the rigours of a second, more extensive, visual field examination.

The induction of visual field defects makes it possible to concentrate on specific types of visual field defects such as those seen in early open-angle glaucoma. Several papers have been published which provide precise information on the location and depth of defects seen in early open-angle glaucoma. ${ }^{25}$ \&

A description of the Friedmann Visual Field Analyser Mark II can be found in the previous paper."

\section{Material and methods}

Three hundred and one normal eyes were examined with the VFA. At the beginning of each examination the patients threshold was established by repeatedly presenting at different intensities $(0 \cdot 2 \log$ unit steps $)$ approximately 30 stimuli. These stimuli were selected to cover the whole $25^{\circ}$ of the visual field tested by the VFA. The threshold was taken as the intensity at which $20-30 \%$ of the stimuli were missed. All stimulus 


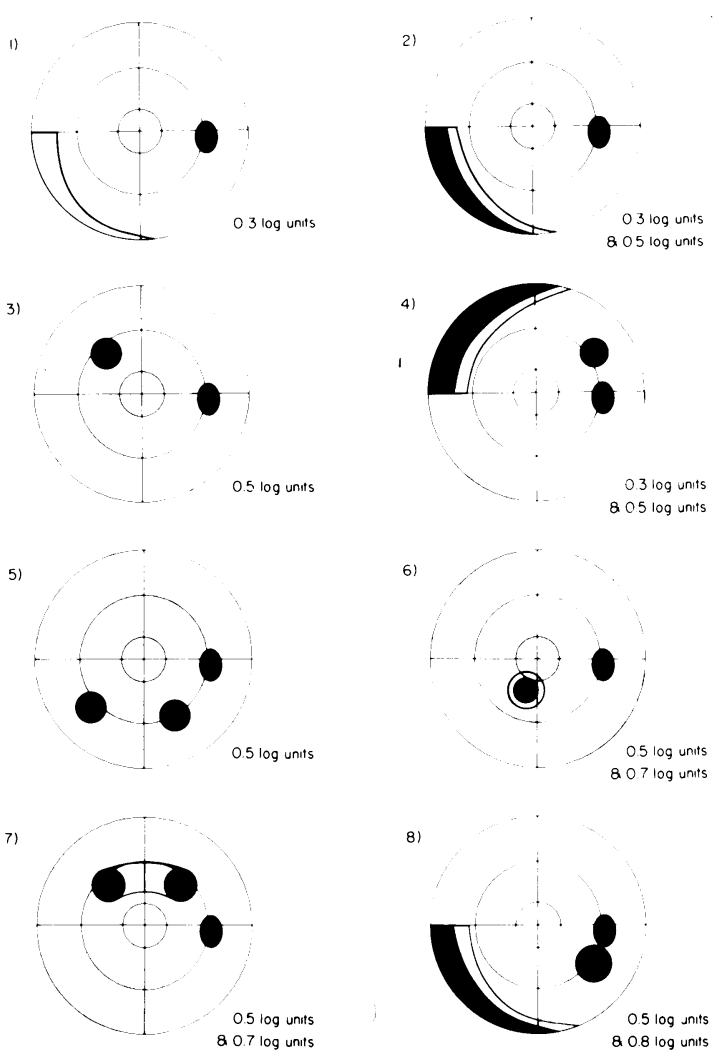

Fig. 1 The position and depth of the induced defects.

patterns were then presented at the threshold value. Any missed stimuli were retested at this intensity; if missed a second time they were then repeatedly tested at gradually increasing intensities ( $(0 \cdot 2 \log$ unit steps) until either they were seen or the upper limit of the instrument's intensity scale was reached.

Visual field defects were induced in 155 of the eyes examined by attaching a circular, transparent sheet, on which small pieces of neutral density filter were attached, to the rear fenestrated plate of the VFA. These filters covered some of the fenestrations in this plate and thereby reduced the intensity of certain stimuli. The number, position, and depth of the neutral density filters were arranged to induce defects similar to those seen in early open-angle glaucoma. Eight different types of defect were induced (Fig. 1). These ranged from an early nasal step (1) to an arcuate scotoma (8). Each defect was presented an approximately equal number of times to an equal number of right and left eyes. The defects numbered (3) and (6), which were both isolated scotoma in the arcuate region, could adopt any one of three positions. In total there were 24 different defects ( 12 for each eye).
At the time of the examination neither the subject nor the perimetrist knew whether a defect had been induced or if it had its location. All the examinations were conducted by the same perimetrist, who was experienced in using the instrument prior to the evaluation.

On completion of the trial the results of each examination were entered into a computer for analysis. The computer kept a record for every subject of the stimuli missed at threshold and, if they were seen at higher intensities, the intensity at which they were seen.

Most of the subjects used in this trial were selected from the students and staff of the University. The majority of them had not previously undergone a visual field examination.

\section{Results}

The results from the 146 eyes in which no defects were induced are given in the previous paper." These results can be used to establish a set of criteria that can then be applied to the group with induced defects. Each set of criteria will have a specificity calculated from the 146 eyes with no induced defects and a sensitivity calculated from the 155 eyes with an induced defect.

Initially a set of criteria slightly modified from those of Batko et al. "'were applied to the data. This involved failing anybody who either; (1) missed a point at 0.8 $\log$ unit above threshold, other than those that fall within the blind spot region, the extreme $\left(>20^{\circ}\right)$ superior field and the one faulty point $23^{\circ}$ to the left and $9.5^{\circ}$ above the fixation point"; or (2) missed a group of 2 or more stimuli at $0.4 \log$ unit above threshold within the central $200^{\circ}$ of the visual field.

These criteria result in $6.8 \%$ of the normal population failing the test and $46 \%$ of the defective population passing it. The high false positive rate can be reduced if the second criterion is changed to missing a group of three or more stimuli. The high false negative rate $(46 \%)$ is largely the result of the defects numbered 1-4. Only $26 \%$ of these were detected, while $85 \%$ of those in categories $5-8$ were positive. In clinical evaluations that rely on populations of patients with early visual field defects it is unlikely that many subjects would have defects as slight as those in categories $1-4$.

An alternative to applying pass/fail criteria is to give each response a score based on the number of points missed, their location, the intensity at which they were seen, and whether or not they were clustered together. The results of one such form of analysis are given in Fig. 2(a). The abscissa is the score obtained and the ordinate the specificity calculated from the 'normal' sample and the sensitivity 
(a)

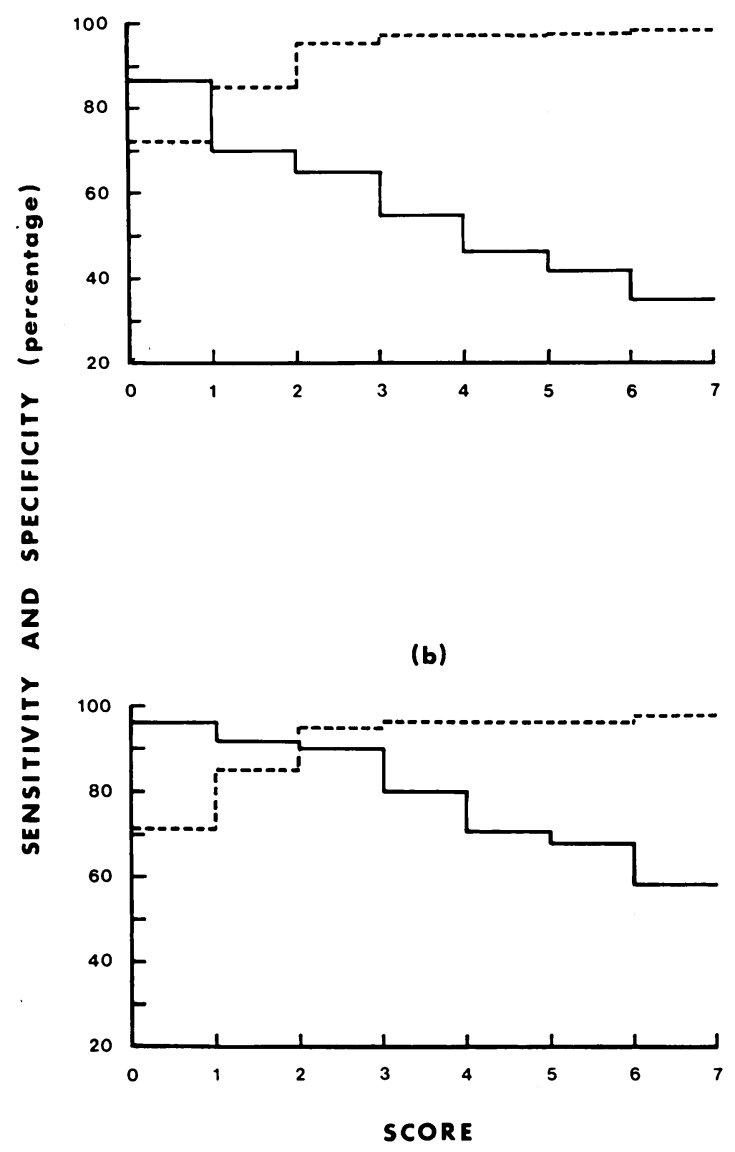

Fig. 2(a) The sensitivity (solid line) and specificity (dashed line) of the Friedmann Visual Field Analyser Mark II versus the field score for all 'normals' and all 'defectives. '(b) The sensitivity (solid line) and specificity (dashed line) of the Friedmann Visual Field Analyser Mark II versus the field score for all 'normals' and 'defectives' falling in groups 5-8.

calculated from the 'defective' sample. In this analysis subjects scored:

I point for every stimulus missed at $(0 \cdot 6$ log unit above threshold 2 points .................. $0.8 \ldots \ldots \ldots \ldots \ldots \ldots$

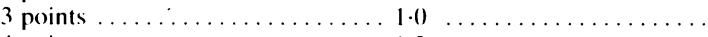
4 points ................. $1 \cdot 2$

2 points for a cluster of 2 missed points at $(0.4 \log$ unit above threshold

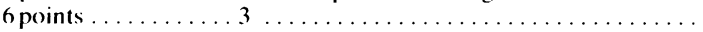

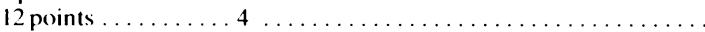
ctc.

The 4 stimuli that fell within the blind spot region" were ignored by the analysis, as was the one faulty point $23^{\circ}$ to the left and $9.5^{\circ}$ above the fixation point. Points were considered to be clustered if they fell within $7 \cdot 5^{\circ}$ of each other.

Examination of Fig. 2(a) reveals that there is con- siderable overlap between the 'defective' and 'normal' groups. No matter what score is chosen as a pass/fail criterion, it always results in either a large number of 'defective' subjects passing the test or a large number of 'normals' failing the test. The results can be made to appear much better by simply removing from the analysis the very early defects. Fig. 2(b) gives the results of the same analysis with defect categories $1-4$ removed. If the analysis is confined to groups 7 and 8 , the instrument becomes, at a score of $8,99 \%$ specific and $95 \%$ sensitive.

The particular form of analysis used to produce Fig. 2 is just one of an almost infinite number that could be applied to the data. Changing the form of the analysis does not, however, have much of an effect on the ability of the VFA to differentiate 'defectives' from 'normals'-that is, the form of analysis used to produce this figure is very close to the optimal one for the data obtained in this study.

\section{Discussion}

The results from this survey indicate that, when a set of criteria which result in a relatively low false positive rate are chosen, the VFA becomes insensitive to early visual field defects. Using the criteria of failing anyone who misses a stimulus at $0.8 \mathrm{log}$ unit or more above threshold and anyone who misses a group of 3 or more stimuli at $0.4 \log$ unit above threshold yields a specificity of $97 \%$ and a sensitivity of $46 \%$. This low measure of sensitivity does not mean that the VFA is any more insensitive than visual field equipment claiming sensitivities in excess of $90 \%$. Measures of sensitivity and specificity depend on the sample chosen. In this evaluation subjects presented themselves with very early induced defects many of which would not have been detected or used in other evaluations. Measures of sensitivity and specificity can be compared with each other only when either they are derived from the same population groups or are derived from duplicated groups of defects. The technique of inducing visual field defects allows us to present exactly the same group of defects with exactly the same frequency on any number of pieces of visual field equipment and therefore allows the comparison of different pieces of equipment and different techniques of examination.

The application of pass/fail criteria to visual field data is of little value to the clinician who has to decide whether to refer or treat a patient for glaucoma. He cannot tell, without going back to the original data, whether the patient just failed the test or has an advanced defect. Similarly, he cannot tell whether the patient has a perfectly normal visual field response or was just below the failing criteria. One solution to this problem is to score each set of data and then 
Fig. 3 An example of how a computer can replot the visual field data using a series of grey scales to denote the depth of any scotoma. The computer also gives an estimate of field survival (bottom left hand corner) and indicates on the vertical scale the field score.

\section{FRIEDMANN MARK II ANALYSIS}

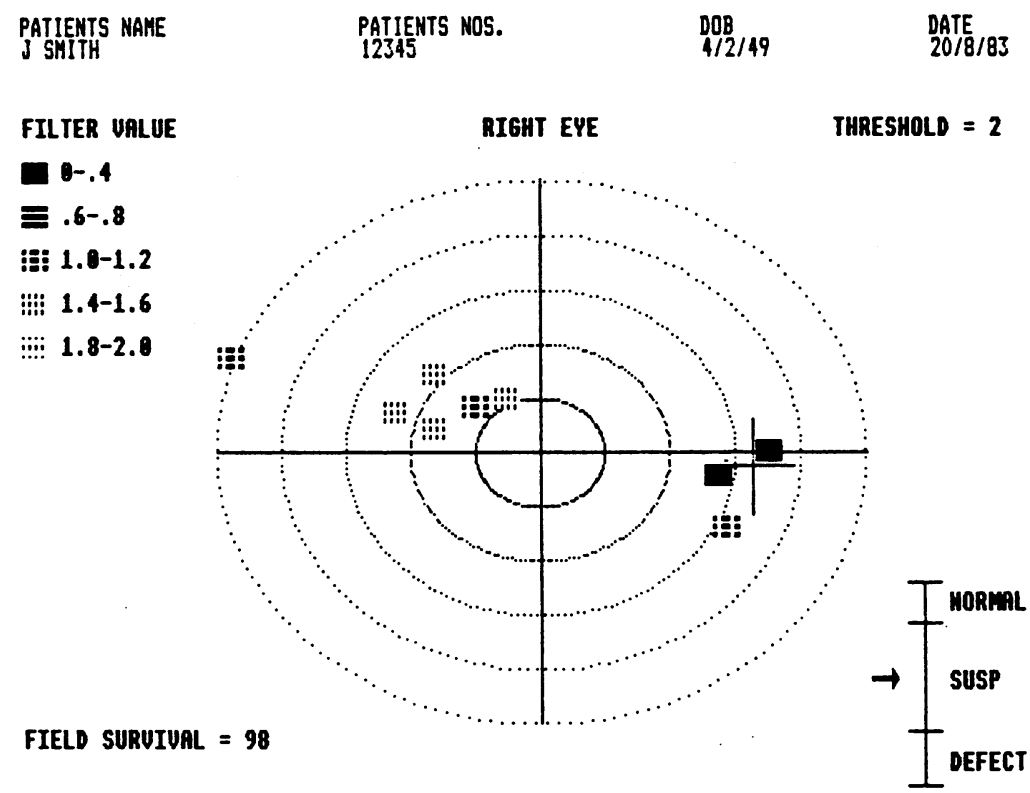

compare the score with those from both 'normal' and 'defective' populations. It would then be possible to state the probability of any given field response being 'normal' or 'defective.' An example of how this particular form of analysis could be used in the clinical situation is given in Fig. 3. The arrow pointing to the vertical scale marked NORM/SUSP/DEFECT gives the score for the data presented. The arrow can adopt any one of 15 positions on the scale. The first 3 fall within the NORM region of the scale and account for $95 \%$ of the 'normals' within this survey. This area of the scale also includes $35 \%$ of the induced 'defectives' (10\% of defect groups $5-8)$. The SUSP region includes $4 \%$ of the 'normals' and $41 \%$ of the induced 'defectives' (38\% of defect groups 4-8), and the DEFECT region of the scale includes $1 \%$ of the 'normals' and $24 \%$ of the induced 'defectives.' By noting the position of this arrow the practitioner can quickly decide his course of action.

Applying scores to visual field data can also be used to evaluate whether a visual field defect is progressing or not. Sponsel et al. used a unit of measure called 'field survival' to monitor the extent of the visual field recorded with a Goldmann perimeter. "12 This measure, which extends from 0 (no field) to 100 (full field) can, when monitored over several patient visits, provide the ophthalmologist with information about the efficacy of any particular form of treatment. Fig. 3 also includes a measure of field survival that can be used for the same purpose. Again it ranges from 0 (no field) to 100 (full field) and is approximately linearly related to the extent of the field.

Many of the current generation of perimeters have internal microprocessors which could easily cope with the type of analysis demonstrated here. Those that do not can easily be linked to small microcomputers that will not only perform this analysis but will often improve the output format of the data. Fig. 3, which is the output from a small microcomputer system, replots the Friedmann data using grey scales to denote the depth of any scotoma. This format overcomes the critisms of De Boer et al., ${ }^{13}$ who stated that the standard examination chart was overcrowded and difficult to interpret. Computer generated plots also overcome problems encountered when perimetrists use different techniques to represent the data. These variations are often so great that they can completely mask any slight differences in actual field configurations.

The technique of field examination used in this survey was designed to obtain sufficient information to allow the selection of an ideal form of analysis. Once this has been decided upon it no longer becomes necessary to adopt such an extensive examination 
routine. The analysis selected within this survey does not take into account the results obtained at threshold and those taken at $0 \cdot 2 \log$ unit above threshold. If this type of analysis were used in a clinical situation, it would be unnecessary to record these data. Once the subject's threshold had been established, the intensity could be turned up by $0 \cdot 4 \log$ unit before testing all the stimuli.

Difficulties in differentiating between the two groups of data has highlighted two ways in which the technique used to measure the visual field with the VFA could be improved. If, as suggested in the previous paper, every missed point was re-examined with the eccentric fixation point, it would have been possible to differentiate between the isolated missed points that are the result of angioscotoma and those that are the result of scotoma which extend more than $2^{\circ}$ from the missed stimulus (the eccentric fixation point effectively displaces all stimuli $2^{\circ}$ in any selected direction). The second modification to the way in which the data were collected which could have led to a better differentiation between the 'normals' and 'defectives' relates to the technique used to establish the threshold. In this survey a series of different stimuli were presented at different intensities $(0.2 \mathrm{log}$ unit steps) until one was found at which approximately $20-30 \%$ were missed. It was not unusual to find subjects who at one intensity setting would miss practically all the stimuli, while at the next higher setting they would see practically all the stimuli. In these cases the threshold lay somewhere between the two settings. Forcing the perimetrist to choose a setting that is either above or below the true value increases the variability of the data. If the perimetrist chooses the setting at which the majority of the stimuli were missed, then he increases the likelihood of a false positive. If he chooses the setting at which the majority were seen, then he increases the likelihood of a false negative. The VFA enables the intensity to be altered in $0 \cdot 1 \log$ unit steps. In addition to the filter control knob that adjusts the intensity in $0.2 \log$ unit steps there is an additional filter wheel that enables a $-0.3 \log$ unit filter to be inserted in the light path (a $+0.3 \mathrm{log}$ unit filter is in fact removed from the light path when the wheel is in the $-0.3 \log$ unit position). Inserting this filter does not, however, alter the digitally displayed filter value, which means that care has to be taken when using this filter so as not to record the wrong value.

\section{References}

1 Greve EL. Performance of computer assisted perimeters. Doc Ophthalmol 1982; 53: 343-80.

2 Greve EL, Verduin WM. Detection of carly glaucomatous damagc. Part 1. Visual ficld examination. Doc Ophthalmol Proc Ser 1976; 14: 103-14.

3 Johnson CA, Keltner JL. Comparative cvaluation of the Autoficld-1, CFA-120, and Ficldmaster model 101-PR automated pcrimcters. Ophthalmology 1980; 87: 777-84.

4 Fricdmann AI. Outline of Visual Ficld Analyser Mark II. Doc Ophthalmol Proc Ser 1980; 22: 65-7.

5 Aulhorn E. Harms $\mathbf{H}$. Early visual ficld defects in glaucoma. In: Lcydhecker W. Glaucoma Tutzing Symposium. 1966. Baslc: Karger, 1967: 151-86.

6 Aulhorn E, Karmeycr H. Frequency distribution in carly glaucomatous visual ficld defects. Doc Ophthalmol Proc Ser 1976; 14: 75-83.

7 Drance SM. The glaucomatous visual ficld. Invest Ophthalmol Visual Sci 1972; 11: 85-97.

8 Coughlan M, Fricdmann AI. The frequency distribution of carly visual ficld defects in glaucoma. Doc Ophthalmol Proc Ser 1981; 26: 345-9.

9 Henson DB, Dix SM, Obornc AC. Evaluation of the Fricdmann Visual Ficld Analyser Mark II. Part 1. Results from a normal population. BrJ Ophthalmol 1984; 68: 458-62.

10 Batko KA, Anctil J, Anderson DR. Detecting glaucomatous damage with the Fricdmann Analyzer compared with the Goldmann perimeter and cvaluation of stercoscopic photographs of the optic disk. Am J Ophthalmol 1983; 95: 435-47.

11 Sponsel WE, Dallas NL, Burbridge L. Visual ficld survival: the response to timolol therapy in open-angle glaucoma. $\mathrm{BrJ} \mathrm{Oph}$ thalmol 1983; 67: 220-7.

12 Sponsel WE, Williams A, Dallas NL, Henson DB. A microcomputer-based glaucoma assessment system designed for clinical ophthalmologists. Res Clin Forums 1983; 5: 25-35.

13 De Bocr RW, Van Den Berg TJTP. Beintema MR, Greve EL, Hoppener J, Verduin WM. The Fricdmann Visual Ficld Analyser Mark II-Technical cvaluation and clinical results. Doc Ophthalmol 1982; 53: 331-42. 